\title{
Research on Impact Characteristics of Inclined Coal-rock Composite body
}

\author{
Yunliang Tan ${ }^{1,}$, Yubao Zhang ${ }^{1, b}$ \\ ${ }^{1}$ State Key Laboratory of Mining Disaster Prevention and Control Co-founded by Shandong Province and the Ministry of Science \\ and Technology, Shandong University of Science and Technology, Qingdao 266590, China \\ aemail: tyll1p@163169.net, bemail:zybsdust@163.com
}

\begin{abstract}
In this paper, the coal-rock composite models with different inclined angles were established using particle flow code, and the impact instability characteristics after unloading were analyzed. In addition, the evolution process of impact energy was studied. The research results show that the strain energy of coal-rock composite model firstly releases linearly, and then releases obeying a power function. What is more, the kinetic evolution process is divided into accelerated impact stage, stable impact stage, and residual impact stage. With the increase of the inclined angle of coal seam, the accumulated maximum strain energy decreases, while the obtained maximum kinetic energy increases. In the same inclined coal seam, the strain energy and the kinetic energy of the roadway in the lower end of the coal seam were larger than that in the upper end.
\end{abstract}

Keywords - coal-rock composite model; impact instability; different inclined angles; evolution of impact energy

\section{I.INTRODUCTION}

Rockburst is one dynamic disaster encountered during underground mining excavations [1-2]. Many kinds of methods are utilized to reveal the mechanism of the rockburst, including laboratory tests, in-situ monitoring and numerical simulation methods [3-5]. Despite decades of research, the mechanical mechanism of rockburst is not well understood, and therefore they are difficult to predict and control.

In the mining engineering, rock burst is a phenomenon of the sudden failure of the composite coal-rock structure [6-7]. Guo et al. [7] carried out experimental and numerical simulation researches on the strength property and failure mechanism of different inclined angles coal-rock combination bodies. Song et al. [9] obtained the factors influencing energy dissipation in the loading failure process of coal and rock with bursting liability mainly include strength, homogeneity, and energy input efficiency. The purpose of this paper is to study the evolution of strain energy and kinetic energy in the impact instability process of coal-rock composite model with different inclined angles using particle flow code.

\section{II.THEORY AND METHODOLOGY}

A numerical model of coal-rock composite body was built by using particle flow code [10-11]. The model with 4.0 $\mathrm{m}$ height and $1.0 \mathrm{~m}$ width is composed of roof rock, coal seam, and floor rock. The rock and coal are simulated using the parallel bond model, and the micromechanical parameters of the model are listed in Table 1. In the test, the upper boundary of model is applied constant load of 40MPa firstly, and then the lateral restraint of right boundary is removed. At the same time, the strain energy and kinetic energy of the composite model are monitored. The thickness of the coal seam is constant, and the angle of the coal seam is variable. In order to study the influence of inclined angle on impact instability of coal seams, the inclined angle is set as $45^{\circ}, 30^{\circ}$, $0^{\circ},-30^{\circ}$, and $-45^{\circ}$, respectively, as shown in Fig. 2.

\section{III.RESULTS AND DISCUSSION}

Strain energy could be accumulated in the coal-rock composite model under the external load. When the lateral restraint was removed, the strain energy that stored in the composite model released intensely, as shown in Fig.3.

From the releasing curves of strain energy (see Fig. 3), it is found that as follows: 


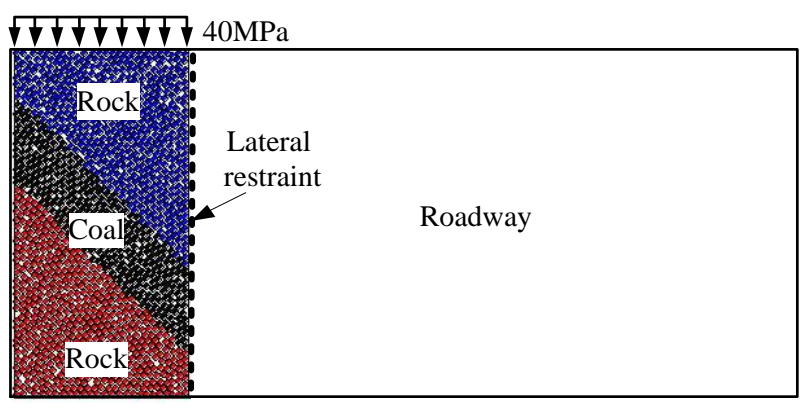

Fig. 1 Model of coal-rock composite body

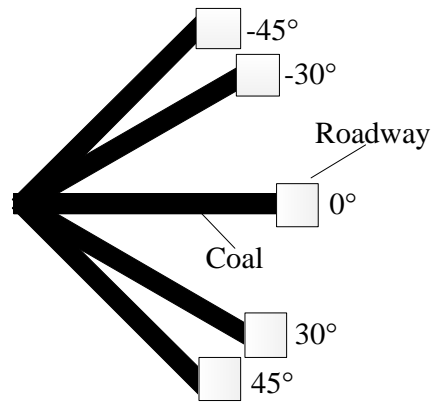

Fig. 2 Schemes of coal seam' inclined angles

Table 1 Parameters of the coal-rock composite model

\begin{tabular}{ccccccc}
\hline Structure & Density $\left(\mathrm{kg} / \mathrm{m}^{3}\right)$ & Radius $(\mathrm{mm})$ & $\begin{array}{c}\text { Friction } \\
\text { coefficient }\end{array}$ & $\begin{array}{c}\text { Bonding radius } \\
\text { factor }\end{array}$ & $\begin{array}{c}\text { Elastic modulus } \\
(\mathrm{GPa})\end{array}$ & $\begin{array}{c}\text { Bonding } \\
\text { strength (MPa) }\end{array}$ \\
\hline Roof rock & 2800 & 5 & 0.5 & 1 & 10 & 50 \\
Coal mass & 2000 & 5 & 0.5 & 1 & 2 & 10 \\
Floor rock & 2800 & 5 & 0.5 & 1 & 10 & 50 \\
\hline
\end{tabular}

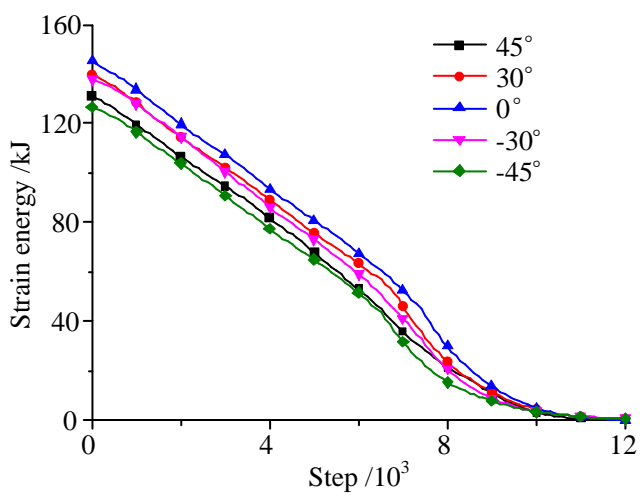

Fig. 3 Curves of strain energy releasing

(1) The strain energy releasing process of the composite model can be divided into two stages. In the linearly releasing stage, the energy releasing rate is great and maintains a constant. In the power function releasing stage, the energy releasing rate gradually decreases, and finally falls to zero.

(2) When the coal seams' inclined angle changes, the curves of strain energy releasing in the model are parallel to each other in linearly releasing stage, and are becoming convergence gradually in power function releasing stage.

(3) As can be seen in Fig. 4, with the increase of the inclined angle of coal seam, the accumulated maximum strain energy in the composite model gradually decreases. Under the same inclined angle, the strain energy of the roadway in the

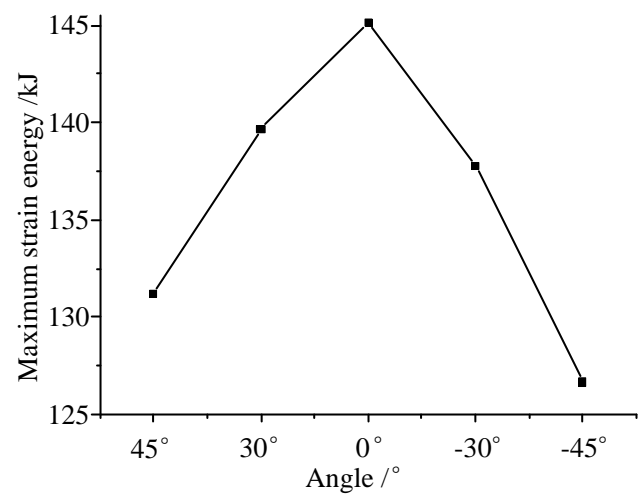

Fig. 4 Maximum strain energy versus inclined angle

lower end of the coal seam is larger than that in the upper end. Therefore, the monitoring of ground pressure should be strengthened in the roadway located in the lower end of the inclined coal seam.

The strain energy of coal-rock composite model can be released in the form of kinetic energy and friction energy, and the kinetic energy has greater influence on the extent of impact damage. The strain energy of composite model releases after removing lateral restraint, and the model particles burst out and obtain kinetic energy. As seen in Fig. 5, it is observed that as follows:

(1) The kinetic energy evolution process is divided into I-accelerated impact stage, II-stable impact stage, and 
III-residual impact stage. In accelerated impact stage, the surface particles of coal- rock composite model get extremely high impact velocities in a short time, and the kinetic energy gradually increases. In stable impact stage, with the gradual release of the strain energy, the impact velocity of model particles decreases gradually, and the total kinetic energy reduces rapidly. In residual impact stage, the strain energy releases completely, and the kinetic energy of particles decreases slowly until it is zero.

(2) With the increase of the angle of coal seam, shear failure easily occurs in the composite model. As shown in Fig. 6 , the evolution gradient of kinetic energy of particles increases gradually and the obtained maximum kinetic energy increases correspondingly. Under the same inclined angle, the kinetic energy of the roadway in the lower end of the coal seam are larger than that in the upper end. Therefore, the safety protection measures should be strengthened in the roadway located in the lower end of the inclined coal seam.

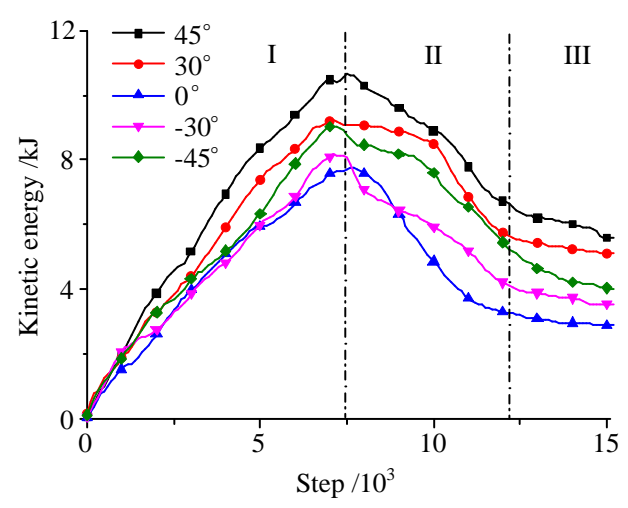

Fig.5 Evolution curve of the kinetic energy

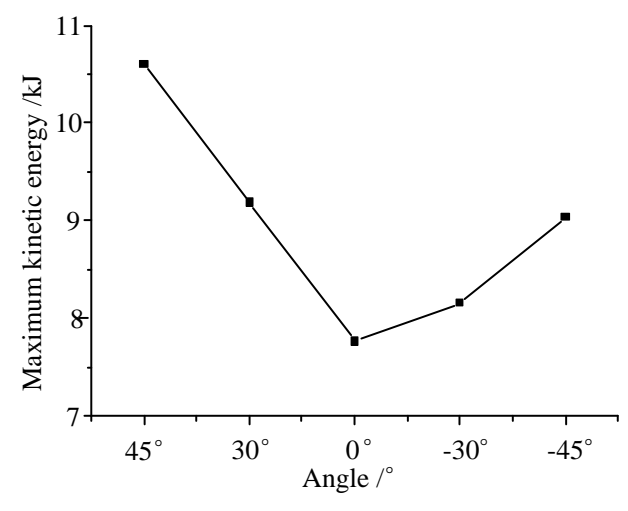

Fig. 6 Maximum kinetic energy versus inclined angle

\section{IV.CONCLUSIONS}

(1) The strain energy release process of coal-rock composite model can be divided into two stages: linearly releasing stage and power function releasing stage. With the increase of the inclined angle of coal seam, the accumulated maximum strain energy in the composite model gradually decreases. Under the same inclined angle, the strain energy of the roadway in the lower end of the coal seam is larger than that in the upper end.

(2) The kinetic evolution process can be divided into accelerated impact stage, stable impact stage, and residual impact stage. With the increase of the angle of coal seam, the evolution gradient of kinetic energy of particles increases gradually, and the obtained maximum kinetic energy increases correspondingly. Under the same inclined angle, the kinetic energy of the roadway in the lower end of the coal seam is larger than that in the upper end.

\section{ACKNOWLEDGMENTS}

This work is supported by National Natural Science Foundation of China (51274133, 51474137), Shandong province science and technology development plan item(2014GSF120002), and Tai'shan Scholar Engineering Construction Fund of Shandong Province of China.

\section{REFERENCES}

[1] Mark Christopher, Gauna Michael. Evaluating the risk of coal bursts in underground coal mines. International Journal of Mining Science and Technology, 2016, 26(1): 47-52.

[2] Iannacchione Anthony T., Tadolini Stephen C.. Occurrence, predication, and control of coal burst events in the U.S.. International Journal of Mining Science and Technology, 2016, 26(1): 39-46.

[3] Yunliang Tan, Yanchun Yin, Shitan Gu, Zhiwei Tian. Multi-index monitoring and evaluation on rock burst in Yangcheng Mine[J]. Shock and Vibration, 2015, Article ID 624893.

[4] Jianchao Wang, Fuxing Jiang, Xiang-jun Meng, Xu-you Wang, Si-tao Zhu, Yu Feng. Mechanism of rock burst occurrence in specially thick coal seam with rock parting, Rock Mechanics Rock Engineering, 2016, 49(5):1953-1965.

[5] Wu Cai, Linming Dou, Guangyao Si, Anye Cao, Jiang He, Sai Liu. A principal component analysis/fuzzy comprehensive evaluation model for coal burst liability assessment. International Journal of Rock Mechanics \& Mining Sciences, 2016, 81: 62-69. 
[6] Yixin Zhao, Yaodong Jiang, Jie Zhu, Guanzhe Sun. Experimental study on precursory information of deformations of coal-rock composite samples before failure. Chinese Journal of Rock Mechanics and Engineering, 2008, 27(2): 339-346.

[7] Bingxiang Huang, Jiangwei Liu. The effect of loading rate on the behavior of samples composed of coal and rock. International Journal of Rock Mechanics \& Mining Sciences, 2013, 61: 23-30.

[8] Dongming Guo, Jianping Zuo, Yi Zhang, Renshu Yang. Research on strength and failure mechanism of deep coal-rock combination bodies of different inclined angles. Rock and Soil Mechanics, 2011, 32(5): 1333-1339. (in Chinese)

[9] Dazhao Song, Enyuan Wang, Zhonghui Liu, Jie Liu, Wenquan Xu. Energy dissipation of coal and rock during damage and failure process based on EMR. International Journal of Mining Science and Technology, 2015, 25(5): 787-795.

[10] Yunliang Tan, Weiyao Guo, Qingheng Gu, Tongbin Zhao, Fenghai Yu. Research on the rockburst tendency and $\mathrm{AE}$ characteristics of inhomogeneous coal-rock combination bodies [J]. Shock and Vibration, 2016, Article ID 9271434.

[11]YIN Yan-chun, ZHAO Tong-bin, TAN Yun-liang, et al. Reconstruction and numerical test of the mesoscopic model of rock based on Otsu digital image processing[J]. Rock and Soil Mechanics, 2015, 36(9): 2532-2540. 